\title{
Lexical Structures for Linguistic Inference
}

\author{
Peter Anick and Sabine Bergler \\ Computer Science Department \\ Brandeis University \\ Waltham, MA 02254-9110 \\ anick@aiag.enet.dec.com \\ sabine@cs.brandeis.edu
}

\begin{abstract}
In order to resolve metonymy and other violations of selectional restrictions between lexical items, a language understander must be able to infer relationships that do not have explicit lexical analogs in the sentence. Although such inferencing has typically been relegated to the world knowledge portion of a natural language processing system, there is also evidence, from both theoretical analysis in compositional semantics and distributional analysis of corpus data, that some cases of metonymy may best be processed with respect to more specific lexical and syntactic constructions. In this paper, we argue how the richer vocabulary for lexical semantics proposed in Pustejovsky's "Generative Lexicon" theory allows one to explore the role of lexical information in such cases, and therefore sheds more light on the distinction between lexical inferences, which follow from defaults associated with lexical items and rules of composition, and pragmatic inferences, which depend on reasoning with respect to the context of the utterance.
\end{abstract}

\section{Introduction}

Is a lexicon merely a set of entry points into a conceptual, "real world" knowledge base, or does it have its own rich structure and rules of inference, related to, yet distinct from the body of world knowledge? In this paper, we explore this question from the point of view of the inferential machinery required to relate a verb and its arguments. We will argue that a rich lexical semantic structure makes it possible to express rules of composition with the degree of precision necessary to account for the many nuances of actual language use, capturing linguistic generalizations based on the semantic content of lexical items rather than relying solely on general purpose inferencing over an encyclopedic knowledge base.

One of the fundamental properties of most computational lexicons is an accounting of the relationship between a verb and its arguments. Typically, each argument position of a verb is annotated with "selectional restrictions" which are intended to constrain the set of nouns which may legally fill the argument position ${ }^{1}$. Such constraints are useful for word sense disambiguation, since semantically anomalous combinations of senses can be readily identified and discarded during parsing. A number of researchers have noted that such selectional restrictions may legitimately be violated under certain circumstances, as in the sentences below:

(1) "The car drank gasoline." ([Wilks, 1978])

(2) "Ted played Bach." ([Fass, 1988])

\footnotetext{
${ }^{1}$ If nouns are organized into a conceptual "isa" hierarchy, then a selectional restriction may be no more than a reference to a position in the hierarchy.
} 
In (1), the "car" violates the selectional restriction that the subject be animate. In (2), the object "Bach" is a person, violating the verb's selectional restrictions (i.e. some form of music). Following [Wilks,1978], Fass [1988] interprets selectional restrictions as preferences and goes on to account for violations of preferences via operations such as metonymy and metaphor. For example, Fass postulates a number of metonymic substitution rules, such as "Artist for Artform", "Container for Contents", and "Part for Whole." When a preference violation is encountered, his algorithm tries applying these metonymic inference rules to the offending noun in an attempt to find the "shortest" coherent path between the verb's preference and some metonymically related concept. Failing this, the algorithm goes on to explore metaphorical interpretations. In a similar vein, [Parisi and Castelfranchi, 1988] make use of a semantic network encoding "encyclopedic" relations among lexical concepts to derive paths between verbs and arguments which violate selectional restrictions. Using a shortest path heuristic, their algorithm interprets the senetence "The pot is boiling." as "The liquid which is contained in the pot is boiling."

While these algorithms work well on a small set of interesting examples, we feel that distance metrics operating on unconstrained encyclopedic knowledge insufficiently characterize the relationship between lexical entries and metonymic inference. In this paper, we present a richer representational framework for lexical knowledge, based on Generative Lexicon theory [Pustejovsky, 1991], and describe a methodology for exploring the interplay between word senses and the metonymic processes they enter into. [Pustejovsky 1991] distinguishes between "logical metonymy", whose resolution falls naturally out of operations on information contained directly in lexical entries, and "pragmatic metonymy", whose resolution depends on interpretation with respect to the proper pragmatic context. We begin with an overview of the major components of Generative Lexicon theory and present data from the semantic domain of musical terms to illustrate how metonymic reasoning is facilitated in a generative lexicon. Then we indicate how in the case of reporting verbs the compositional semantics of the matrix verb and the subject produce pragmatic constrains on the complement. We conclude that it is advantageous to represent the conceptual knowledge that bears on the linguistic behavior of words in the lexicon.

\section{Generative Lexicon}

Pustejovsky's Generative Lexicon theory [Pustejovsky, 1991] rejects the characterization of a lexicon as a static listing of word senses and focuses instead on elucidating the set of generative devices which give rise to word senses and allow for the flexible and creative use of language.

Generative Lexicon theory postulates a number of representational subsystems for the description of lexical items and the rules of syntactic and semantic composition. These include, in addition to argument and event structures, (1) a qualia structure, which partitions the aspects of a noun's meaning into formal, constitutive, agentive, and telic roles, or qualia, (2) a lexical inheritance network reflecting the same partition, (3) Lexical Conceptual Paradigms, which describe sets of syntactic behaviors which correspond to lexical semantic categories, and (4) generative devices for extending the logical senses of lexical items dynamically, as for instance coercion rules, mentioned later in this paper. By attempting to tease apart various aspects of the lexical semantics, the theory provides a new vocabulary with which to approach traditional linguistic problems.

\section{Qualia}

- Constitutive Role: Relation to constituent parts.

- Formal Role: Distinguishes the word within a larger domain. 
- Telic Role: Purpose and function.

- Agentive Role: Whatever brings it about.

To illustrate the use of qualia structures for handling selectional restrictions, consider three schematic qualia structures for food-related nouns:

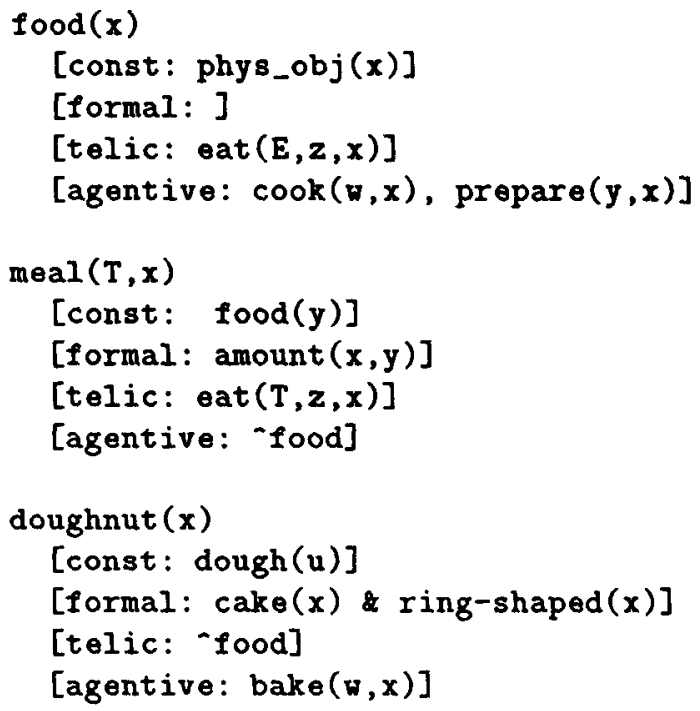

The empty formal role in the structure for "food" indicates that food is a mass noun and, as the constitutive role indicates, composed of some physical material. The telic role is doing most of the work of defining food as something which is eaten. The agentive role adds that food typically arises by cooking or preparing processes (although this is certainly defeasible).

Doughnut also inherits the telic quale from food, but differs from food in that it specifies a value in its formal quale. That is, it "packages" the mass noun in a specific way (cf. Pelletier and Schubert [1986] , Krifka [1987] ). This in fact, is the structural semantic characterization of a packaging coercion: taking a lexical structure with no formal role, and specifying the formal role (Pustejovsky [1991] for details).

Now consider the representation for meal. A meal directly denotes both the event pertaining to the activity of eating the food, as well as the food itself. The fact that meal is defined with respect to food is made evident in the fact that the formal role is a certain packaging of an amount of food, while the telic role includes the event denoting the occasion of the eating. Now consider the following sentences making use of these nouns.

1. John ate the doughnut.

2. John ate the meal.

3. John left after the meal.

4. John left after the doughnut.

The first problem presented by such data is how to describe the selectional restriction imposed by the verb eat on its direct object, since it clearly allows for both physical objects and events. The 
qualia structure suggests a straightforward answer. Instead of stating the restriction in terms of a formal or constitutive property, we may use the telic role. That is, eat may take as direct object any noun whose telic role is eat. This may at first seem like a circular definition, but why should the telic role be any less accessible to the selecting verb than, say, the formal role? This approach also renders it unnecessary to create an explicit subclass of physical objects called edible-objects. The class is implicitly defined via the telic quale.

The next problem is how to accomodate doughnut as the object of a preposition selecting for an event. This sentence requires an inference (i.e. that John left after eating the doughnut) that can only be verified in the discourse context. However, the lexical structures do provide a means for quickly identifying the potential interpretation.

Pustejovsky [1989, 1991], has proposed a system of type coercion to explain how verbs like want and believe can accept arguments of type $S, V P$, and NP without requiring multiple verb entries in the lexicon. He defines type coercion as "a semantic operation that converts an argument to the type which is expected by a function, where it would otherwise lead to a type error." This principle of type coercion can be applied to coercing an object into an event, as required for our doughnut. The telic and agentive roles identify readily available, definitional events associated with a nominal. In most of the examples of this kind of selectional restriction violation that we have encountered, it is the telic role that fills the expected event.

By examing what systematic semantic relationships exist in the grammatical expressions of lexical items, we hope to apply the principles of the generative lexicon to new data and illustrate new phenomena in support of the view of the "non-static" lexicon. Making use of data from human interest "notes" conferences, Wall Street Journal and Time magazine articles, our methodology is to analyze a set of semantically related verbs with respect to their argument types in order to ascertain how the lexical semantic structure of the verb relates to the range of potential (e.g. metonymic) concepts that may appear in an argument position. ${ }^{2}$ We consider first a number of verbs in the domain of music, such as "play", "perform", and "strum." Then we turn to verbs of reporting, as found in newspaper and magazine articles.

\section{Hierarchical classification and qualia structure}

One question is how to assign the definitional information to the respective qualia. While it is beyond the scope of this paper to adequately address this question, let us suggest that the very structure of the definitions of words often suggests which qualia are of relevance. Consider for example a faceted classification scheme (cf. Vickery [1975]), as one might do for a subject matter indexing task for the music domain (the data presented here is based on an electronic "notes" conference on music):

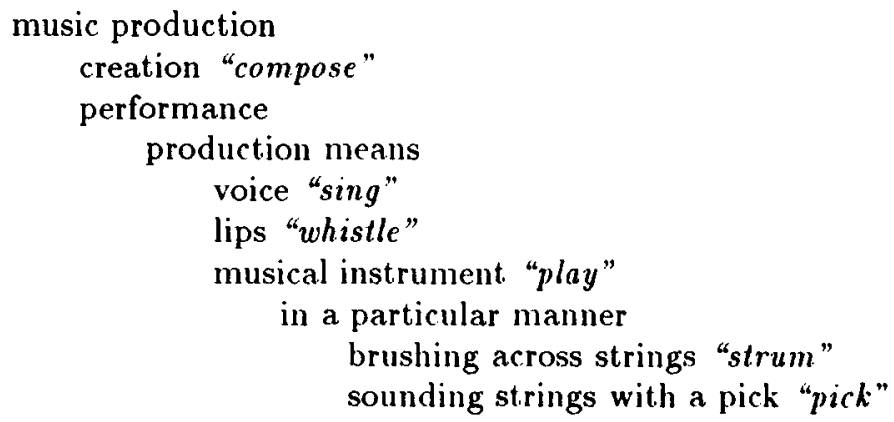

${ }^{2}$ We have made extensive use of Levin's [1990] verb classification, and the methodology of verbal diathesis therein. 


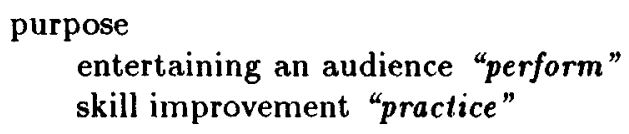

The category "production means" describes, for example, verbs that incorporate different "tools" for making the music, i.e. sing incorporates in its lexical meaning the use of voice, whistle the use of lips and play the use of an instrument. Note that play does not specify the instrument, it implies an instrument but can take as an argument a specification of the particular instrument. Neither sing nor whistle can take an instrument as argument. The implied argument pertains to the constitutive role.

Perform and practice, both purposeful activities by definition, define that purpose in their telic role. Both words select for an event in object position. An interesting difference between these two words is the selectional restriction on the event type for the object position: perform selects for the result of an accomplishment, practice for an activity. This fact explains a difference in metonymic behavior that we will sketch below.

\subsection{Possible violations in object position}

We can arrive in a similar manner at a categorization of nouns in object position of verbs from our musical domain:

\section{Music "packagings"}

unit tune, song, melody, piece, chord, lick, note, repertoire, arrangement

musical form jig, hornpipe, symphony, sonata

relation to other performers lead, backup

location in a piece ending, intro

number of perfomers solo, duet

physical presentation line, measure, bar, page

musical instruments guitar, piano

musical instrument parts string, key, soundboard, bridge

genres $j a z z$, blues

events containing music gig, concert, job, festival, wedding

locations where music is played bar, club, concert hall

composers Bach, Beethoven

instrument makers Fender, Martin

This faceted classification together with data analysis suggests that "music packagings" tune, song, ... are related to packagers [Pelletier and Schubert 1989], in fact "packaging" the mass term music into events. This category can be further refined into "accomplishments" (sonata, piece, ...) and "achievements" (chord, note, ...). This corresponds with the behavior of perform and practice:

to practice a chord

to practice a high note

to perform a sonata

to perform a piece

This discussion gives only a very rough first impression on the workings of the structures of the Generative Lexicon theory when it comes to violations of selectional restrictions. We showed, however, that it is not impossible to characterize the common sense knowledge that pertains to 
linguistic behavior. This is an important goal for a generative lexicon, as it aims to capture exactly that body of knowledge that bears on the linguistic behavior of words (in context) in a structured way, thus constraining common sense inferencing at an appropriate level of granularity.

\section{Lexical constraints on common sense reasoning}

In the previous section we have shown how to identify and categorize the conceptual or world knowledge that directly bears on the syntactic behavior of verbs towards their direct objects in the musical domain. Let us now consider another semantic field, namely reporting verbs in the newspaper context. These verbs frequently occur with a metonymy in subject position. We show how the violations of the selectional restrictions can be dealt with in the Generative Lexicon framework, producing at the same time a pragmatic coherence constraint for the complement clause.

Most utterance verbs can be used in reported speech in newspaper articles, selecting for two arguments, namely a proposition as complement clause and a source as subject. The interpretation of these instances of reported speech is that the journalist quotes or reports the proposition in the complement clause giving as evidence for its credibility or reliability the original source of the reported material. (cf. Bergler, forthcoming)

Two examples of reported speech in newspaper texts are:

A Lincoln spokesman said its management "never authorized or participated in any bugging of anyone."

-Wall Street Journal, 10/27/89

He said "I have not misled anyone" about US policy in Iraq.

-Boston Globe, $4 / 5 / 91$

Reporting verbs are pervasive in newspaper articles [Bergler, 1991]. The sense of an utterance verb as a reporting verb is sometimes slightly different from its literal sense. We therefore introduce a semantic type REPORTING-VERB, which allows us to define a small part of the lexical hierarchy of reporting verbs as follows:

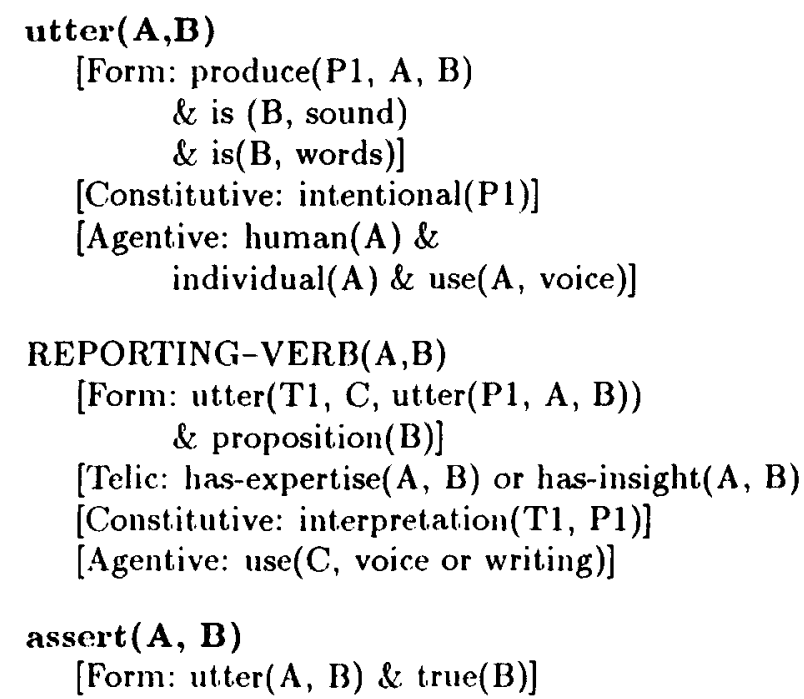


$\operatorname{say}(A, B)$

[Form:

[Literal: utter(A,B)]

[REPORTING-VERB: $\operatorname{assert}(A, B)]$ ]

announce $(A, B)$

[Form: $\operatorname{say}(T 1, A, B)]$

[Constitutive: new(B)]

[Agentive:

legitimation( $\mathrm{A}, \mathrm{T} 1)$
$\operatorname{claim}(A, B)$

[Form:

[Literal: $\operatorname{assert}(\mathrm{A}, \mathrm{P1}) \& \operatorname{own}(\mathrm{P1}, \mathrm{A}, \mathrm{B})$;

demand(T1, A, B)]

[REPORTING-VERB: assert(A, B)

$\&$ opposition $(\psi, \mathrm{B})]$

\section{$\operatorname{insist}(A, B)$}

[Form: $\operatorname{claim}(A, B)$

[Constitutive: MANNER: vehement]

This partial hierarchy is a very crude classification of the four reporting verbs say, announce, claim, and insist. We have indicated a literal word sense where it differs significantly, but have made no attempt to define the respective lexical entries completely. Note that the semantic type REPORTING-VERB specifies the basic "reporting situation", especially that the telic role (roughly describable as its "purpose") is that the source has expertise on the topic of the complement clause or at least some (relevant) insight.

As reported in Bergler [1991], we find that reporting verbs frequently occur with different kinds of metonymy. The violated selectional constraint is that the source of the utterance, i.e. the agent, is not a single individual human being, but a city or institution or even a building, as illustrated below:

1. Marlin Fitzwater said ...

2. Washington said ...

3. The White House said ...

4. IBM announced ...

All reporting verbs accept metonymic extensions where the original human source is replaced by some significant superclass they fall into. In the case of reporting verbs of special importance is the employer $(I B M)$ or the membership in a group (the French). But we find also the characterization of the source in terms of a role he or she played, as in the witness. This "knowledge" can best be stored in the concept of REPORTING-VERB - all the verbs defined with a reporting verb sense will then inherit the "permission" to use metonymic extensions to a superclass or a specific role played in an event.

But this is not a sufficient restriction on the possible metonymies. Consider the following scenario: a German makes a disparaging remark $\phi$ about the German government in the United States.

The types of expected descriptions of the source, that would be deemed appropriate, might be the following:

Hans Glueck said that $\phi$ (Name)

A German said that $\phi$ (Nationality)

? A Mercedes Benz employee said that $\phi$ (Affiliation)

however, given what $\phi$ denotes the following descriptions would be deemed inapproprite and in fact uninformative (cf. Grice [1967], Gazdar [1979] )

A human said that $\phi$

A vegetarian said that $\phi$

A European said that $\phi$ 
This example illustrates that not all true descriptions of a source can be used as subject in reporting contexts. The source has to be relevant to the topic of the complement clause. Bergler [forthcoming] describes that the lexical realization of the source in newspaper articles generally adds to the evaluation of the credibility or reliability the source has with respect to the topic. Thus a German in general has more competence and thus reliability on issues concerning the German government because of direct affectedness; this fact however is lost if we characterize him (correctly) as a European.

This is little surprising; general pragmatic considerations such as Gricean maxims ("Be relevant", "Be informative") account for this fact. It has been noticed that it is extremely difficult to put these pragmatic considerations into computational form [Wilks, 1986]. We will show here that some pragmatic coherence can, however, be established on the basis of lexical semantics.

When analyzing the subjects for all occurrences of seven reporting verbs in a corpus of 250,000 words from the TIME Magazine (1963), we found that a small semantic grammar described the different lexicalizations of the subject, moreover, that grammar shows that the source is often lexcalized as some institution or as holding a particular position. It is beyond the scope of this paper to discuss why this should be so; suffice it to allude to the fact that newspapers and magazines report very frequently on government activity and business developments and that insider information is considered more valuable.

The dilemma here is that a basically pragmatic problem is part of the selectional restrictions of reporting verbs and that therefore the occurring systematicity has to have some reflection in a lexical semantics. What does the Generative Lexicon solution to this problem look like?

Let us first state the problem with an example. Announce selects for a subject that has some "legitimization" for making the statement that is repeated or paraphrased in the complement. The relation between subject and complement that constitutes the "legitimization" is established by the projective conclusion space, a device that generates certain semantic relationships between lexical items dynamically, much in the way a semantic net records semantic relationships between words statically. ${ }^{3}$

The lexical definition of officialis in the American Heritage Dictionary is:

official adj

1. Of, pertaining to, or authorized by a proper authority; authoritative.

2. Formal or ceremonious: an official banquet.

n

1. One who holds an office or position.

2. A referee in a sport.

and in the Longman Dictionary of Contemporary English we find:

official adj of or about a position of trust, power, and responsability: an official position/an official occasion/an official manner of speaking. - opposite: unofficial; compare officious

which results in a GL entry of the form ${ }^{4}$

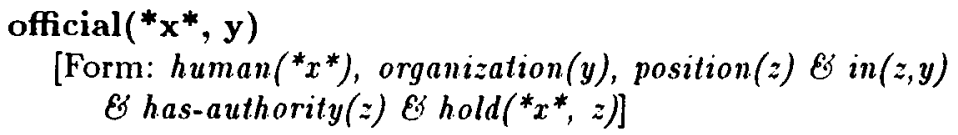

\footnotetext{
${ }^{3}$ For detail, see [Pustejovsky 1991]
}

1 see [Bergler, forthcoming] for detail. 
[Telic: work-for $\left.\left({ }^{*} x^{*}, y\right), \operatorname{trust}\left(y,{ }^{*} x^{*}\right)\right]$

[Constitutive: individual $\left({ }^{*} x^{*}\right)$ ]

[Agentive: $\Uparrow y$ ]

The combination Officials announced sounds very appropriate (semantically "comfortable") regardless of the content of the complement clause, because the semantic selectional restriction imposed by announce is met at the lexical semantic level by the definition of official as somebody of authority or trust, implicitly "legitimized". This is why Officials announced sounds more like a phrasal pattern than like an ad hoc generative combination.

This is an obvious case where a simple feature matching procedure can establish the coherence relation given two lexical entries. Consider the slightly more complex case of Chrysler officials announced $\phi$, which introduces constraints on the content of the complement, restricting it to matters related to the automobile industry in general. Chrysler officials is a particularization of officials in general and is thus more specific. A more specific source has a more specific domain of expertise (and is thus often more reliable). This means that the lexical coherence between Chrysler officials and announce is established just like above with the additional constraint for the complement to be coherent (i.e. to relate to something about) Chrysler.

Lastly, the metonymic use of Chrysler announced $\phi$ causes a violation of the selectional restriction of announce, which leads to a coercion of Chrysler to an equivalent of Chrysler officials ${ }^{5}$.

The interpretation process of the three subject NP phrases Officials, Chrysler officials, and Chrysler in the context of announce is entirely due to the lexical semantics of the words involved, and has thus far not involved random common sense inference, which is one of the main ideas behind GL (namely to minimize common sense reasoning to the really hard cases).

Let us now make the leap to say, a reporting verb that has no explicit semantic constraints. We know that anybody can say anything without in fact being of much consequence. The use of say as a reporting verb in the newspaper context, however, restricts its use to statements where the source has some insight or experience that justifies repeating or rephrasing his/her words. This is a fact related to the concept of REPORTING-VERB, in fact an anchor for coercion.

The case of Officials said $\phi$ is analog to the case of Officials announced $\phi$, with officials being in a position of authority or trust filling the requirement associated with the concept REPORTING-VERB, an inherited and therefore weaker constraint than that for announce. Similarly, Chrysler officials said that $\phi$ derives the coherence relation from the concept REPORTING-VERB and constrains the topic of the complement clause to coher with Chrysler.

Chrysler said $\phi$ is more marked than Chrysler announced $\phi$, but indeed does occur frequently, for example in the Wall Street Journal corpus. Interestingly, we found that in the sentences that contained a company name as subject of say, the complement phrase was frequently referring back to the subject with the pronoun it as in:

- AEG also said it expects group operating profit to remain at last year's level of 115 million marks.

- Chantal said it is in advanced stages of testing one of the drugs, Cyoctol, as a topical treatment for mild to severe acne.

- And Nissan Motor Co., reacting to foreign pressure on Japanese auto makers, said it plans to slash annual vehicle exports in half by the late 1990s.

- San Miguel said in a report to its stockholders that higher wages, production costs, and interest rates threaten growth prospects for the second half.

\footnotetext{
${ }^{5}$ We expect the process at work here to be very close to that of ellipsis resolution.
} 
This enhances the markedness of the sentences enforcing the same coercion based on a stipulation of metonymy as for announce. But note that it is possible to use say in its reporting sense with a much weaker coherence relation between the source and the complement than the one required for announce.

We have derived coherence relations between three different source NPs, Officials, Chrysler officials, and Chrysler to two reporting verbs, namely announce and say from the lexical semantics of the words involved and we have shown how the compositional behavior of the matrix clause in turn introduces topic constraints on the complement. This means that an issue as inherently pragmatic sounding as restricting the metonymy in subject position of reporting verbs to extensions that bear relevance to the complement clause can partly be resolved on the basis of lexical semantics, when treating the lexicon not as a static, passive list of definitions but a dynamic structure that can incorporate procedures that were previously only found in common sense reasoning systems. The important advantage to pure common sense reasoning systems lies in the fact that the reasoning in GL is constrained by and limited to syntactic and lexical semantic knowledge, incorporating conceptual knowledge only where it bears on language behavior. This results in much cheaper inference mechanisms, but will not explain the truly creative use of language.

\section{Conclusion}

In this paper we considered violations of selectional restrictions for verbs of different semantic fields. First, we discussed musical terms as they occur in an electronic "notes" conference and found that violations of the selectional restriction for the direct object (restricted to "music") in fact describe a systematic set of "aspects" of music, indeed allowing us to formulate coercion rules that make it possible to speak of the required semantic inferences in general terms. Coercion rules are a generative device, illustrating one of the dynamic aspects of the Generative Lexicon formalism, which aims to represent world knowledge that is reflected in the syntactic and semantic behavior of words. The inference rules used in a generative lexicon are reminiscent of common sense reasoning but much more tightly constrained (and therefore limited).

We illustrated on the musical terms that interesting differences in the acceptance of violations of the selectional restrictions for the object position can be represented (and explained) by abstractions that often hold in other domains. For instance we confirmed that the notion of "packager", occurring with mass terms in general, plays an important role in the musical domain as well.

This approach is advantageous compared to purely syntactic/semantic or purely common sensical approaches because it identifies certain pragmatic considerations needed to describe syntactic/semantic behavior (notoriously missing or assumed in syntactic or formal semantic theories) yet constrains the immense space for pragmatic inferences according to the lexical definitions and a small set of generative devices, thus leaving to common sense reasoning proper only truly novel uses of language.

The semantic domain of reporting verbs served to illustrate that not only can we resolve selectional violations within this paradigm, but that from the very compositional meaning of a reporting verb with its subject, we can automatically derive pragmatic constraints on the topic of the complement clause, thus limiting even here the work of the pragmatic inferencer.

\section{Acknowledgements}

We gratefully acknowledge James Pustejovsky for his invaluable help with this paper. 


\section{References}

[Ber91] S. Bergler. The semantics of collocational patterns for reporting verbs. In Proceedings of the Fifth Conference of the European Chapter of the Association for Computational Linguistics, Berlin, April 9-11, 1991, 1991.

[Berng] S. Bergler. Evidential Analysis of Reported Speech. PhD thesis, Brandeis University, forthcoming.

[Fas88] D. Fass. An account of coherence, semantic relations, metonymy, and lexical ambiguity resolution. In S. Small, G. Cottrell, and M. Tanenhaus, editors, Lexical Ambiguity Resolution: Perspectives from Psycholinguistics, Neuropsychology, and Artificial Intelligence. Morgan Kaufmann Publishers, San Mateo, 1988.

[Gaz79] G. Gazdar. Pragmatics: Implicature, Presupposition, and Logical Form. Academic Press, New York, 1979.

[Gri67] H.P. Grice. Logic and conversation. Unpublished MS. of the William James Lectures, Harvard University, 1967.

[Kri87] M. Krifka. Nominal reference and temporal constitution: Towards a semantics of quantity. In Proceedings of the Sixth Amsterdam Colloquium, pages 153-173, University of Amsterdam, 1987.

[levng] B. Levill. Towards a Lexical Organization of English Verbs. University of Chicago Press, Chicago, forthcoming.

[PC88] D. Parisi and C. Castelfranchi. Disambiguation in a lexically based sentence understanding system. In S. Small, G. Cottrell, and M. Tanenhaus, editors, Lexical Ambiguity Resolution: Perspectives from Psycholinguistics, Neuropsychology, and Artificial Intelligence. Morgan Kaufmann Publishers, San Mateo, 1988.

[PL86] F.J. Pelletier and L.K.Schubert. Mass expressions. In D. Gabbay and F. Guenthner, editors, Handbook of Philosophical Knowledge, Vol. 4. Reidel, Dordrecht, Holland, 1986.

[Pus91a] J. Pustejovsky. The Generative Lexicon: A Theory of Computational Lexical Semantics. MIT Press, Cambridge, 1991.

[Pus91b] J. Pustejovsky. Towards a generative lexicon. Computational Linguistics, 17(3), 1991.

[Vic75] B.C. Vickery. Classification and Indexing in Science. Butterworth and Co., London, England, 1975.

[Wil78] Y.A. Wilks. Making preferences more active. Artificial Intelligence, 10:1-11, 1978.

[Wil86] Y.A. Wilks. Relevance and beliefs. In T. Myers, K. Brown, and B. McGonigle, editors, Reasoning and Discourse Processes, pages 265-289. Academic Press, New York, 1986. 